\title{
Bluetooth Tracking Approach For User Assistance Based In Sequential Patterns Analysis.
}

\author{
Aitor Arribas Velasco \\ Dublin Institute of Technology, aitor.arribasvelasco@tudublin.ie \\ John McGrory \\ Technological University Dublin, john.mcgrory@tudublin.ie \\ Damon Berry \\ Technological University Dublin, damon.berry@tudublin.ie
}

Follow this and additional works at: https://arrow.tudublin.ie/archastrocon

Part of the Electrical and Electronics Commons, and the Engineering Education Commons

\section{Recommended Citation}

Velasco, A., McGrory, J., \& Berry, D. (2019). Bluetooth tracking approach for user assistance based in sequential patterns analysis. IHSED: 2nd International Conference on Human Systems Engineering and Design: Future Trends and Applications Universität der Bundeswehr München, Munich, Germany September 16-18. doi:10.21427/8geg-jj23

This Conference Paper is brought to you for free and open access by the Archaeoastronomy Research at ARROW@TU Dublin. It has been accepted for inclusion in Conference Papers by an authorized administrator of ARROW@TU Dublin. For more information, please contact arrow.admin@tudublin.ie, aisling.coyne@tudublin.ie,gerard.connolly@tudublin.ie.

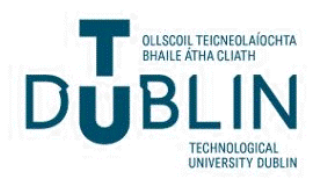




\title{
Bluetooth tracking approach for user assistance based in sequential patterns analysis.
}

\author{
Aitor Arribas Velasco ${ }^{1, *}$, John McGrory ${ }^{1}$, Damon Berry ${ }^{1}$ \\ ${ }^{1}$ Technological University Dublin, School of Electrical and Electronic engineering, TU \\ Dublin, Kevin Street, Dublin 8, D08 NF82. \\ \{Aitor.ArribasVelasco, John.Mcgrory,Damon.Berry\}@dit.ie
}

\begin{abstract}
As a civilization, we are drowning in a raging torrent of data, of our own making, that is being harvested by our collective technologies and systems (e.g. Fitbit, phones). However, data itself is of no utility unless it is converted into beneficial knowledge. Design patterns have been shown to be a pragmatic solution to control and manage information flows and provide order and meaning to data within a given context. Assisting users within their daily activities has become a key aspect for modern Artificial Intelligence Systems. Nevertheless, although the GPS technologies work well for outside location, indoor positioning is still problematic, while is a vital awareness component in ambient assistance. This paper shows a preliminary Bluetooth tracking system with a focus on the user's transition between areas of interest. Our work aims to shed light on how the term design patterns can be applied for studying human behavior patterns in the smart environment.
\end{abstract}

Keywords: Bluetooth low Energy - Design Patterns - Smart Environments Presence Detection.

\section{$1 \quad$ Introduction}

A pattern is an entity that, when repeated in a sequence, contributes to guide or solve common reusable design issues [1]. They exist in different contexts such as nature, art and architecture, computer science and so on, allowing humans to mathematically model natural processes. For example; patterns, as observed in the natural environment, represent the optimization of a process [2].

Nowadays, technology has considerable impact on the use of indoor environments and the interaction of humans inside buildings such as houses or public spaces. Emerging technologies such as the Internet of Things (IoT) allow urban spaces to be designed in order to improve the experience of users while they are carrying out daily activities. IoT has therefore become an essential tool for enabling smart environments [3], with which systems can directly interact and assist users. Currently, IoT systems are developed and installed in a wide range of scenarios, such as smart homes or smart cities, in other words, enhancing the smart-living experience [4]. 
In line with both urban design and software design, the term design patterns has established the possibility of sharing and reusing general solutions to commonly occurring and repeatable problems within a given context. Thus, research has been carried out on these lines in order to optimize the design of new smart spaces [5]. However, a common reusable framework to identify and analyse the sequence of activities followed by users in the smart environment has not yet been defined [5].

Hence, we present a new design and implementation of a Bluetooth based tracking system, for both outdoors and indoors user's transitions between spaces, and not just focused on the location aspect. We combine the transition component to location and activity aspects to get an increase level of granularity related to the context. This paper relies on previous theoretical research published [6], which sought to lay the foundations for a technology-independent design pattern format.

Therefore, our work attempts to anonymously observe activity patterns and so provide guidance and insights into "where, what and who" a rule set based on the "design pattern" format and how this format could contribute to describe a general "understanding" of given cases in the smart environment domain, as well as allowing different processes to collaborate with each other. Applying in areas such as assistive technology and environmental monitoring [7].

\section{Related Work}

To illustrate the complexity of the environment Fig. 1 shows a domestic setting example. For ease, the different spaces are shown in a simplified version above the building. In this example, an Item (e.g. coffee, cooker), Activity (e.g. make coffee, cook in oven or cook) and Location (e.g. the location of the room from a map perspective) are highlighted, but many more aspects can exist.

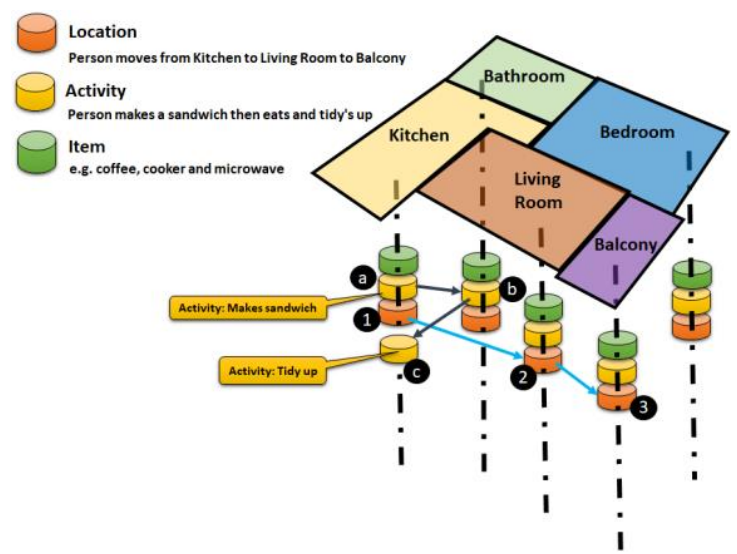

Fig. 1. Image showing vertices linking between nodes.

In Fig. 1 two scenarios are depicted illustrating transitions between locations aspect of each room. In this example, a person is moving from (1) Kitchen to (2) Living room 
to (3) Balcony. Hence, we are tracking their movements. Also shown on the same image are activity transitions aspects were a person is (a) making a sandwich, shown as a kitchen specific activity to (b) eating the sandwich, shown here as a living room (perhaps in front of TV) to (c) tidying up, which is a kitchen specific activity. These are two different node networks and the system can become very complex very quickly. The linking of nodes is not novel, the extracting of the context is.

However, although the term design patterns have been widely used to explain, predict or facilitate decision making, mathematical and computational models also have limitations. Furthermore, there is currently no pattern based system to cover smart spaces that is specifically designed for representing assistive technology problems in domestic, professional care and occupational therapy situations.

Our work focuses on how IoT technology can assist users with specific emphasis on providing context for particular user cases of daily activities, such as, independent living of elderly population. In particular, focusing on the transitions between locations within user activities in smart spaces, and understanding points in time when guidance may be needed.

\section{Methodology}

Design patterns and its related mathematical models have been used in a multitude of areas with different purposes, mainly to facilitate the work of designers [8]. From our current point of view, the study of design patterns is a view to use anticipation to optimize the decision-making based on previous experience. In other words, if I can see what is happening over the time and I can identify the pattern form of that sequence, then I can interact directly in the process in order to assist when an alteration occurs. This approach could also be applied to understanding the behaviour of users who are involved in different processes in the wide variety of smart public spaces, such as a home, museum or hospital.

\subsection{Description of the Elements of the System}

The core elements of our tracking system comprises two key components, an ARMbased embedded system, BBC Micro Bit, worn by the user and an array of detectors, and a set of Raspberry Pi 3, which are fixed in place at different areas of interest through the space. The first device, BBC Micro Bit, is being chosen due to its range of sensors and capability to work as a Bluetooth Low Energy (BTLE) peripheral [9]. These properties provide us with valuable data such as acceleration of the user, hence, being able to determine if the user is moving, standing upright, or he or she has fallen. On the other hand, the Raspberry Pis [10] which have on-board BLE transceivers, acted as anchor nodes receiving the RSSI signal from the BBC Micro Bit carried out by the user. 


\subsection{Description of the Implementation of the System}

The system is described as follows, by following the steps represented in Fig. 2.

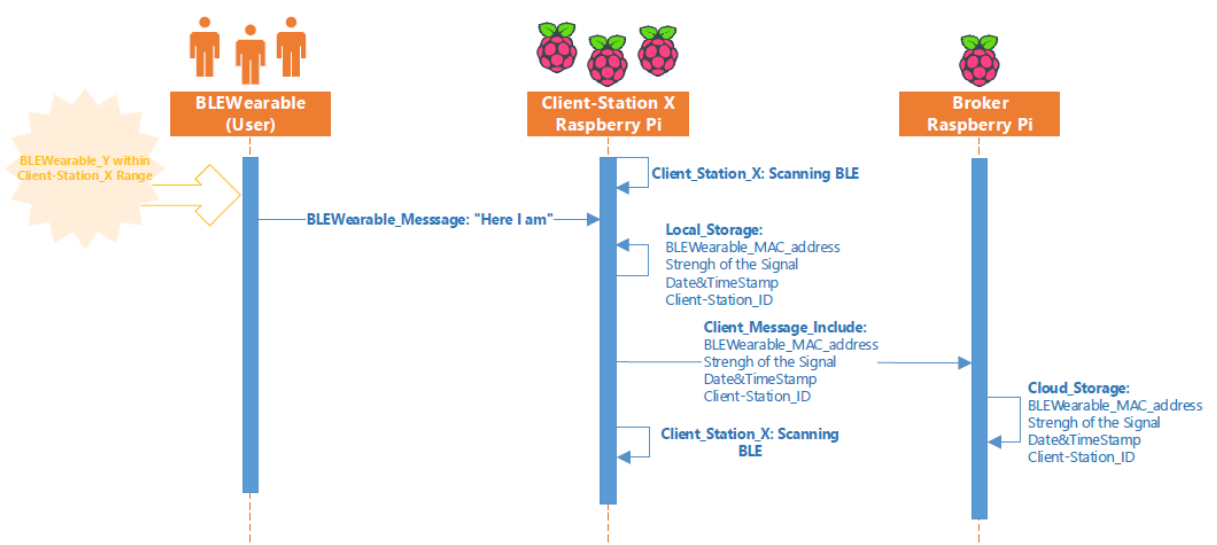

Fig. 2. First-part of the implemented system.

This project seeks to understand how citizens move in civic spaces. It necessarily involves gathering information which could disadvantage citizens if the information is not collected a manner that minimises data protection concerns. Therefore we have decided to adopt a policy of anonymisation at source where that is possible.

With the objective of preserving user anonymity, our system involves a technique called hash encoding that comprises of taking a string of characters such as "sesame 11:22:33:44:55:66" and turning it into a unique and very long number. Two of the interesting features of hash encoding is that each unique string always produces the same very long unique number / identifier, and also that the process is one way, once you have the number it is almost impossible to go backwards and get the original string. These two properties when taken together make the technique very useful for anonymization. The most popular hashing algorithm at the moment is the SHA algorithm and SHA-2 (the one selected for this study) is considered to be suitably secure.

It enables the movements of an individual user to be studied without recording mac address which could be related back to the identity of the user if the mac address was discovered independently. Furthermore, our source code will only record and store the hash encoded mac address of the BTLE peripherals used and described in the above section, BBC Micro Bit.

Hence, the BLEWearable device carried out by the user is constantly broadcasting a Bluetooth signal same as a lighthouse emits a white light. When the BLEWearable_Y is within a Client-Station_X range, this one, first, will locally store 4 data elements for every BLEWearable broadcast by a passers-by BBC Micro Bit. These four elements are: Hashed Mac Address, Received Signal Strength Indicator (RSSI), Timestamp (date and time), and the Client-Station_ID for the measurement. The RSSI parameter allows the system to estimate the distance to and, therefore, the current position of the 
user in a building. In relation to this last parameter, and in order to increase the precision of each measurement, we calculate the average of three values detected. In other words, each RSSI measurement recorded correspond to the average of three RSSI values scanned by the same station. We have implemented this feature, as multiple Client-Stations located in different rooms will pick up simultaneously the same BLEWeareble_Y, but this one, can only be in one place at the same time. Therefore, only an average greater that $-80 \mathrm{~dB}$ will consider that an user is actually in that location. This value, $-80 \mathrm{~dB}$, is the result of multiple tests where standing $2.5 \mathrm{~m}$ away of the Client-Station-X we measured this average RSSI values, and so, allowing us to consider an user in a particular location.

Then, once a Client-Station_X has stored the previous data mentioned, this one will be sent to a data warehouse, or Broker. This last interaction allows us to gather all information from the multiple Client-Stations into a common database, as well as to back up the data recorded. Our system is being coded to handle multiple connections simultaneously, therefore, being able to track multiples peripherals at the same time.

\section{Preliminary Results and Discussion}

Our objective is not necessarily to reach conclusions but rather to prompt new ideas of how design-pattern techniques can be applied in the smart environment domain. Thus, our first scrip coded provides the following valuable information.

1. Number of transitions realized or edges.

2. Number of Client-stations or nodes.

3. For each node, the incoming and outgoing edges.

4. The number of times that a particular transition has been done. For example, this information will allow us to know how many times a user has transited between two particular locations.

This information could be used to identify possible pattern interactions between areas of interest and by, for example, in a museum, this information could provide insights into how the visitors move through the gallery, allowing our system to provide and suggest more personalized touring information, or save time from busy areas.

\section{Conclusion and Future Work}

A multitude of researches have shown how design patterns techniques can enhance the labour of designers, being of particular importance in computer science [11]. Thus, these entities are seen as a consolidated solution to a recurring problem. However, they are not popular in the recognition and analysis of human interaction in traditional householders. Our system attempts to identify those sequences within the known smart environment domain. Where if we describe each of the elements in- 
volved either in a process or in a sequence of activities, we begin to open the door to cross-compatibility and then to collaboration. This information will allow us to:

1. Identify similar behavioural patterns repeated over time by different users.

2. Assist users in real-time in case that an unexpected event or emergency occurs. For example, in a construction building context or assisting an elderly patient living on his/her own.

3. In public spaces, such as Museums, Airports or Hospitals, the behaviour pattern form of the users could contribute to managing patient waiting lines, thereby optimizing services and reducing patients' waiting time.

In addition, our research focuses on the transitions between spaces and how that could affect the original design and future proofing it through universal design concepts. Therefore, by exposing these Location Nodes, Activities and Transitions we can provide much more evidence-based education to the designers and installers of the future.

This research aims to provide an easy installation and configuration so over time evidence based information could be gathered to highlight the optimum design within the space. To conclude, in addition to recording and analyzing users transitions in a range of different smart spaces, our goal is to set a design patterns based platform able to predict and provide a basis for elderly assistance, decision making and guiding as well as understanding human behavior in the smart environment domain.

\section{References}

1. "The Free Dictionary: Patterns." [Online]. Available: http://tinyurl.com/ybwug9lc. [Accessed: 02-Sep-2018].

2. P. Ball, Shapes: Nature's Patterns: A Tapestry in three Parts. Oxford University Press, USA.

3. D. J. Cook and S. K. Das, "How Smart are our Environments? An Updated Look at the State of the Art The Role of Physical Components in Smart Environ ments," J. Pervasive Mob. Comput., vol. 3, no. 2, pp. 53-73, 2007.

4. C. Gomez, S. Chessa, A. Fleury, G. Roussos, and D. Preuveneers, "Internet of Things for enabling smart environments: A technology-centric perspective," J. Ambient Intell. Smart Environ., vol. 11, no. 1, pp. 23-43, 2019.

5. M. Vega-barbas, I. Pau, and J. C. Augusto, "Interaction Patterns for Smart Spaces : A Confident Interaction Design Solution for Pervasive Sensitive IoT Services," no. November, 2017.

6. A. A. Velasco, J. Mcgrory, and D. Berry, "Patterns within Patterns within the Smart Living Experience," 2018, pp. 1-5.

7. D. Lea, "Christopher Alexander: An Introduction for Object-Oriented Designers," ACM SIGSOFT Softw. Eng. Notes, vol. 19, no. 1, pp. 39-46, 1994.

8. A. Dearden and J. Finlay, "Pattern languages in HCI: A critical review," Human-Computer Interact., vol. 21, no. 1, pp. 49-102, 2006.

9. "BBC micro : bit MicroPython Documentation," 2016.

10. R. Krishnan, "Introduction to Raspberry Pi," Control, no. April, p. 2014, 2014.

11. A. Ampatzoglou, "Research state of the art on GoF design patterns: A mapping study," J. Syst. ..., vol. 86, no. 7, pp. 1945-1964, 2013. 